\title{
Numerical Simulation of the Infrared Spectra of Thin Organic Films
}

\author{
A. C. Tenório and C. P. de Melo \\ Departamento de Física \\ Universidade Federal de Pernambuco \\ 50.670-901 Recife PE Brazil
}

Received 08 September, 1998

\begin{abstract}
We present results of numerical simulations of the infrared spectra of thin films of behenic acid (b.a.) in different spectroscopic configurations. A model of independent oscillators was used to derive the optical constants of the material in the infrared region and, from these, the reflectance expressions corresponding to several experimental situations. In this manner, the theoretical attenuated total reflection spectra could be fitted to the experimental result through a steepest-descent routine, and the spectroscopic parameters so optimized were used in the numerical computation of the behavior of the optical constants and of the different spectra of interest. We present results for both Langmuir-Blodgett thin films and thicker evaporated samples of b.a. deposited on ZnSe crystals. In the former case, we also analyze the best suited experimental configuration for the thickness estimate of the films. We have shown that the preliminary use of numerical simulation techniques can simplify the investigation of the spectroscopic properties of thin organic films by allowing the operating limits and capabilities of each experiment to be estimated. Since fatty acids multilayers are the prototypal organized molecular structures, we expect that these techniques become specially important in the spectroscopic investigation of molecular order in thin organic films.
\end{abstract}

\section{Introduction}

Fatty acids can be considered as the prototypical amphiphilic molecules, i.e. those which exhibit (usually at opposite sides) the simultaneous presence of polar and apolar groups ${ }^{[1]}$. When a fatty acid is dispersed in aqueous medium the competition among entropic and energetic contributions to the interaction between solvent and solute molecules can lead to the spontaneous formation of complex structures such as micelles, vesicles and bilayers ${ }^{[2]}$. In special, if a diluted solution of a fatty acid is carefully spread at the air-water interface, a stable floating monolayer can be formed; under appropriate lateral compression this monolayer becomes increasingly rigid and organized and can eventually be transferred to a solid substrate. This constitutes the basis of the so-called Langmuir-Blodgett (LB) technique, introduced in the first half of this century ${ }^{[1]}$. In the last two decades this old technique has experienced a revival, as organized films of polymers and biological molecules have become materials of crescent interest in the fields of Biology, Chemistry, Physics and Electronics $[3]^{\prime}[4]$.

On the other hand, in the last few years selfassembly (SA) techniques have been developed as a competitive method for obtaining the controlled molecular deposition of organic polymers ${ }^{[4]}[5]$. A selfassembled film can be prepared by successive immersions of an adequately treated substrate in alternate recipients containing solutions of appropriate poly-ions. While different examples of complex SA films of conjugated polymers have, for instance, found application as active layers in light-emitting devices ${ }^{[6]}$ and gas sensors ${ }^{[7]}$, it is still an open question the comparison of the degree of order of these structures relative to those of equivalent LB films.

In spite of its importance, both for the basic understanding of the microscopic processes in these films and from the practical point of view, the control of the molecular order in these structures remains an elusive goal in the field. For instance, in a very organized thin 
film of a conjugated polymer one could hope to maximize the macroscopic electronic and optical response of the sample. Also, as phospholipid bilayers can be used to mimic biological membranes, the details of possible interactions of different drugs with these structures could be better investigated if organized mixed LB films of these compounds are produced ${ }^{[8]}$.

At the same time, as an independent development, in the last decade a rapid progress in different variations of infrared (IR) spectroscopic techniques have established that a careful analysis of the IR spectrum of a compound can not only allow the identification of the individual chemical groups present in the molecule but, also, makes possible to determine their relative spatial orientation $^{[9]}$. As a consequence, in our lab we have initiated a program of using Fourier Transform Infrared (FTIR) techniques ${ }^{[10]}$ to investigate the molecular order in thin organic films ${ }^{[11]^{\prime}[12]}$. To the advantages of low cost and simplicity, FTIR experiments combine the exquisite sensitiveness required to assess the relative orientation of characteristic molecular groups present in these organized thin films.

In recent years, numerical simulation techniques have appeared as a convenient tool of investigating the limits of applicability of different spectroscopic experiments. Based on the optical theory, these methods rely on numerical procedures to adjust the reflectance and absorbance expressions of a sample in a given experimental configuration to the corresponding experimental spectra ${ }^{[13]}$. From this, the optical constants of the material are determined and its spectra in other experimental geometries can be theoretically generated.

In this paper we show that the search for the FTIR geometry best suited to the investigation of the spectroscopic properties of thin organic films can be simplified by the preliminary use of numerical simulation techniques to estimate the operating limits and capabilities of each experiment. After a first IR spectrum of a given film is obtained using one of the available FTIR configurations, simple physical models for the infrared active molecular vibrations can be adopted to theoretically reproduce this spectrum by a best-fitting procedure. With this, the appropriate set of spectroscopic parameters describing the characteristic peak structure is determined and one can directly calculate how the index of refraction and the absorption coefficient of the sample vary in the corresponding range of frequencies. Also, use of the Fresnel equations ${ }^{[14]}$ for the different geometries of each IR experiment provide a simple way to generate the corresponding IR spectrum as a function of the sample thickness. In this manner one can preview the results of different FTIR experiments even before they are actually performed.

We begin by reviewing the basic operating principles of each FTIR experiments to be used, and comment about the Fresnel equations obeyed in each case. Then, after a brief review of the steepest-descent minimization algorithm, we provide examples of how the numerical simulation of FTIR spectra can be used to plan the best sequence of experiments of characterization of thin organic films.

\section{FTIR Experiments}

FTIR techniques are convenient tools for the characterization of organic compounds, since they could be easily adapted to the investigation of gaseous, liquid and solid samples ${ }^{[10]}$. Also, different FTIR geometries can be used in the surface analysis of films: for instance, in the case of internal reflection studies (see below) of thin films deposited on an appropriate substrate, the presence of an evanescent field propagating along the interface between the two media offers an unique way of collecting data of even a single monolayer of the material ${ }^{[15]}$.

The standard geometry for an IR spectroscopic investigation corresponds to the transmission mode, in which the infrared radiation reaches the detector after crossing the sample at a normal angle. Because of this, transmission experiments have limited use in the investigation of LB or SA films, since for this configuration the plane where the electric field intensity is maximum is usually perpendicular to the direction of the principal axis of symmetry in these organized structures. For this reason, in the following discussion we will consider only the cases of external reflection ${ }^{[16]}$ and internal reflection experiments ${ }^{[5]}$. In all cases we will assume that the sample has an index of refraction $n_{2}$ and an absorption coefficient $k_{2}$.

\subsection{External reflection mode $(\mathrm{erm})$}

A reflectance spectrum is obtained when the infrared radiation is incident on the surface of the sample accordingly to a (non-vanishing) angle $\Phi$ (see Fig. 1). In the case of a smooth surface, the signal to noise relation (SNR) of an external reflection experiment is relatively high since most of the radiation will eventually 
reach the detector. This fact makes the $\mathrm{erm}$ the preferential choice for the analysis of high absorptive samples, systems for which a successful transmission experiment would require the use of extremely thin specimens.

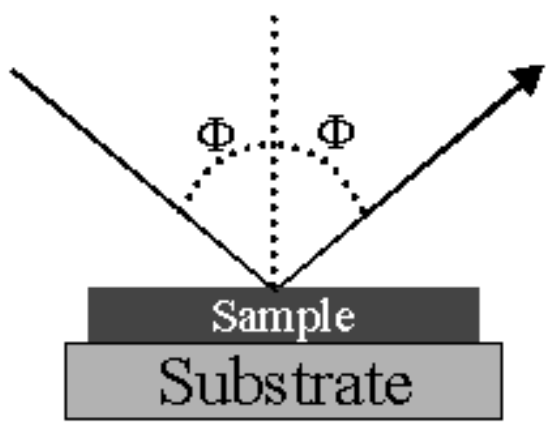

Figure 1. Optical arrangement for the spectroscopy in the external reflection mode. (A metallic coverage is usually applied to the substrate to improve the SNR in grazing angle experiments.)

The reflectance spectrum, which will be determined by the incidence angle $\Phi$ and the optical constants $n_{2}$ and $k_{2}$ of the material, will carry information about the absorption peaks of the sample. Although the interpretation of the corresponding spectra is more complicated than those of transmission experiments, as we will discuss later - see Section III - optical theory can be used to resolve the specific details ${ }^{[17]}$.

The reflectance $R$, the physical quantity measured at the detector, can be calculated from the Fresnel equations ${ }^{[14]}$, which express the boundary conditions obeyed by the Maxwell equations at the interface between the two media with indices of refraction $n_{1}$ and $n_{2}$ (in the present case, air and the material sample, respectively). These boundary conditions are different for the components of the electric field along the directions parallel $(\mathbf{P})$ and perpendicular $(\mathbf{S})$ to the plane of incidence ${ }^{[18]}$. As a consequence, the corresponding reflectance expressions

$$
R_{\|}=\left|\frac{n_{2}^{2} \cos \Phi-n_{1} \sqrt{n_{2}^{2}-n_{1}^{2} \sin ^{2} \Phi}}{n_{2}^{2} \cos \Phi+n_{1} \sqrt{n_{2}^{2}-n_{1}^{2} \sin ^{2} \Phi}}\right|^{2}
$$

and

$$
R_{\perp}=\left|\frac{n_{1} \cos \Phi-\sqrt{n_{2}^{2}-n_{1}^{2} \sin ^{2} \Phi}}{n_{1} \cos \Phi+\sqrt{n_{2}^{2}-n_{1}^{2} \sin ^{2} \Phi}}\right|^{2}
$$

exhibit characteristic and distinct dependences with $\Phi$ (see Fig. 2). As it can be seen from Fig. 2, there is a specific incidence angle $\Phi$ in which the reflected wave is naturally polarized; this is the Brewster angle, $\Phi_{B}=\arctan \left(n_{2} / n_{1}\right)$. Also note that at large values of
$\Phi$, the total reflectance approaches its maximum value. This latter configuration corresponds to the grazing angle (ga) spectroscopy ${ }^{[19]}$, a convenient tool for the investigation of highly absorbing samples.

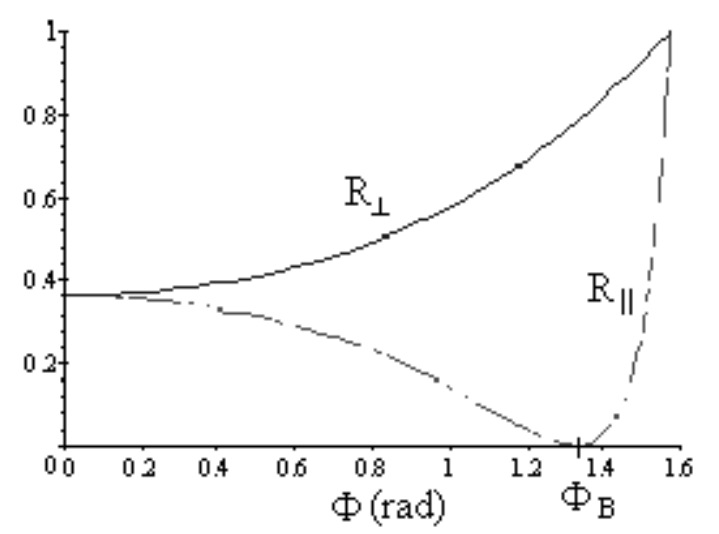

Figure 2. Parallel and perpendicular reflectances in the external reflection mode for the case $n_{1}=4.03$ and $n_{2}=1.0$. Note the existence of the Brewster angle $\Phi_{B}=1.3276 \mathrm{rad} \approx$ $76^{\circ}$ for the parallel polarization.

For the case of thin samples, the presence of a reflective element with optical constants $n_{3}$ and $k_{3}$ besides offering the necessary physical support for a usually fragile specimen - can be used to expand the range of applications of the erm. For instance, in situ experiments can be performed to collect the reflectance spectrum of a floating monolayer in an airwater interface ${ }^{[20]}$. On the other hand, if a metallic surface is used as the supporting substrate of the thin film the technique is sometimes called reflectanceabsorbance ( $\mathrm{ra}$ ); in this case, the metal, characterized by a plasma frequency ${ }^{[21]}$

$$
\varpi_{p}=\left(\frac{N q^{2}}{\varepsilon(\omega \rightarrow \infty) m}\right)^{1 / 2}
$$

(where $\varepsilon(\omega \rightarrow \infty)$ is the high frequency dielectric constant, $q$ and $m$ are respectively the charge and mass of the electron, and $N$ is the electron density per volume), acts as an effective mirror for the IR radiation. Since a higher SNR can then be obtained ${ }^{[22]}$, this becomes a convenient scheme for the investigation of chemical reactions occurring at the interface.

Because of the small penetration length of the IR radiation in metals, even a simple metallic coverage of the reflective element suffices to eliminate the component of the electric field normal to the incidence plane (and therefore parallel to the surface) from the reflected wave, which then becomes $\mathbf{P}$-polarized. This special 
situation makes the $g a$ technique very adequate to the investigation of structural anisotropies in thin films; we have applied the method to the study of the relative orientation of different molecular groups in LB films of conducting polymers ${ }^{[23]}$ and of retinal molecules ${ }^{[11]}$.

\subsection{Internal reflection mode $(\mathrm{irm})$}

Attenuated total reflection ( $A T R$ ) is the standard configuration (see Fig. 3) of the irm, when a sample of index of refraction $n_{2}$ is deposited on the surface of an infrared element (ire) of index of refraction $n_{1}>n_{2}$. Since the IR radiation propagates along the more dense medium before reaching the interface, there exists a critical angle value

$$
\Phi_{c}=\arcsin \frac{n_{2}}{n_{1}},
$$

for which total reflection occurs and an evanescent wave is propagated along the interface. If the sample has an absorption coefficient $k_{2}$, for incidence angles $\Phi \geq \Phi_{c}$ the total reflection is attenuated due to the interaction of the evanescent wave with the material: when, after multiple reflections at the interface, the radiation is finally collected by the detector it carries information about the normal vibrational modes of the sample. Since the reflected wave propagates along the more dense medium, the reflectance expressions (Eqs. (1)-(2)) remain valid, but now, due to the fact that $n_{1}>n_{2}$, they correspond to complex quantities ${ }^{[15]^{\prime}}[18]$. In Fig. 4, we show how the parallel and perpendicular reflectances vary with changing incidence angles $\Phi$.

The penetration length [24]

$$
d_{p}=\frac{c}{\omega\left(\sin ^{2} \Phi-n_{21}^{2}\right)^{1 / 2}},
$$

(where $\omega$ is the angular frequency of the radiation and $\left.n_{21}=n_{2} / n_{1}\right)$, measures the distance transversed by the evanescent wave into the sample before its intensity decays to $1 / e$ of its value at the interface. Two different limits can then be identified for the $A T R$ spectroscopy: (i) thin films, for which the thickness $d \ll d_{p}$, and (ii) thick films, when $d \gg d_{p}$. In the case of thin films, reflection of the wave at a second interface between the sample and a third medium of index of refraction $n_{3}$ must be considered ${ }^{[18]}$. Due to the interference between the two contra-propagating waves in the interior of the sample, the corresponding value of the reflectance $R$ exhibits an oscillatory behavior as a function of the thickness $d$.

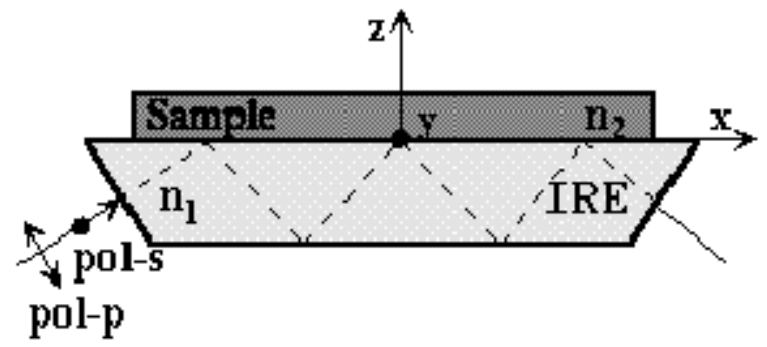

Figure 3. Optical arrangement for the spectroscopy in the internal reflection mode.

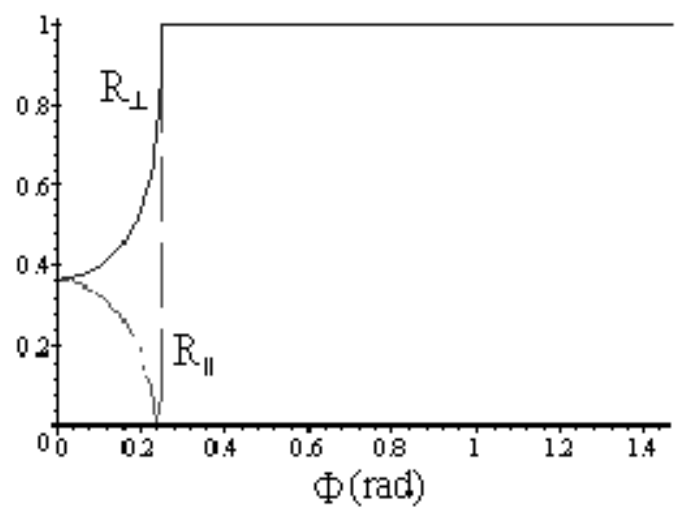

Figure 4. Parallel and perpendicular reflectances in the internal reflection mode for the case $n_{1}=1.0$ and $n_{2}=4.03$. Note that total reflection occurs at angles greater than the critical value of $0.251 \mathrm{rad} \approx 14.4^{\circ}$.

The ire must be transparent to the IR radiation; also, a good contact between the sample and the ire at the interface is an essential requisite for the technique. We have adopted the ATR configuration for the investigation of the molecular order of LB films of conducting polymers directly deposited on a ZnSe crystal ${ }^{[12]}$.

In the following section we will review how the molecular vibrations can be described by a simple model of independent damped harmonic oscillators.

\section{Modelling the Molecular Vi- brations}

In the study of the infrared spectrum of a molecular system, as a convenient first approximation the molecular vibrations can be considered as a collection of independent harmonic oscillators, the normal modes ${ }^{[25]}$. When an external electromagnetic field $\vec{E}$ is applied upon the molecule, each one of these oscillators of mass $m$, charge $q$ and frequency $\omega_{n}$ becomes polarized. If an intrinsic damping factor $\gamma_{n}$ proportional to the velocity is assumed, the solution

$$
\vec{r}_{n}(\omega)=-\frac{q / m}{\omega_{n}^{2}-\omega^{2}-i \omega \gamma_{n}} \vec{E}
$$


of the corresponding equation of motion permits the moment per volume unit) of the medium as calculation of the polarizability (i.e. the total dipole

$$
\vec{P}(\omega)=N \sum_{n} f_{n} q \vec{r}_{n}(\omega)=\vec{E} \sum_{n} \frac{N f_{n} q^{2} / m}{\omega_{n}^{2}-\omega^{2}-i \omega \gamma_{n}},
$$

where $N$ is the electron density per volume unit and $f_{n}$ is the oscillator strength of the vibration transition of resonance frequency $\omega_{n}$.

After introducing the radiation wavenumber $\bar{\nu}=\omega /(2 \pi c)$ and considering the relationship between the dielectric constant $\varepsilon$ and the complex index of refraction $\hat{n}=n+i k$, one can write ${ }^{[13]^{\prime}[17]}$

$$
(\hat{n})^{2}=1+\frac{\frac{N \alpha_{0}}{\varepsilon_{0}}+\frac{N q^{2}}{4 \pi^{2} c^{2} \varepsilon_{0} m} \sum_{n} \frac{f_{n}}{\bar{\nu}_{n}^{2}-\bar{\nu}^{2}-i \bar{\nu} \gamma_{n}}}{1-\frac{N \alpha_{0}}{3 \varepsilon_{0}}+\frac{N q^{2}}{4 \pi^{2} c^{2} 3 \varepsilon_{0} m} \sum_{n} \frac{f_{n}}{\bar{\nu}_{n}^{2}-\bar{\nu}^{2}-i \bar{\nu} \gamma_{n}}},
$$

where the term $N \alpha_{0} / \varepsilon_{0}$, resulting from the electronic contribution $\alpha_{0}$ to the polarizability, was in an ad-hoc manner added to the numerator. Because of its physical origin, this term is independent of the IR frequency.

We must call attention to the fact that for a incoming radiation of wavenumber $\bar{\nu}$, the index of refraction depends on the electronic polarizability $\alpha_{0}$ of the material and is a function of the set $\left\{f_{n}, \gamma_{n}\right\}$ of simple vibrational parameters. Numerical minimization algorithms can then be used to extract these parameters by a procedure of best-fitting this function to known experimental values of the intensity at each characteristic absorption frequency $\bar{\nu}_{n}$. In the next section we will consider how this can be achieved.

\section{Numerical Simulation of the IR Spectra of Thin Organic Films}

In a given reflection experiment the reflectance $R$ can be related to the optical constants $n$ and $k$ of the sample and of the ire, and one can use the corresponding spectrum with characteristic wavenumbers $\bar{\nu}_{n}$ to determine the values of the set of parameters $\left\{f_{n}, \gamma_{n}\right\}$. Once this set of parameters associated to the relevant fea- tures of the IR spectrum of the material is identified, the behavior of the optical constants $n$ and $k$ can be determined. Since the optical constants are independent of the experimental method used, the reflectance $R$ for any other experiment can be theoretically predicted with basis in the expressions derived from the Fresnel equations for that specific configuration ${ }^{[13]}$. Before presenting a few examples of the use of this scheme to investigate the spectroscopic properties of thin organic films, we will first briefly review a simple numerical procedure for best-fitting a theoretical expression to a given set of experimental data points.

\subsection{The steepest-descent minimization proce- dure}

A best-fitting procedure has the goal of finding the values of a set of $M$ parameters $\theta_{k}$ which best adjust a generic function $y=f(x, \theta)$ to $N>M$ known values $y_{i}$. After an initial guess for the $\theta_{k}$ parameters is introduced, the error $d_{i}=y_{i}-f(x, \theta)$ can be determined for each of the data points. One can minimize the function $D=\sum_{i=1}^{N} d_{i}^{2}$ by searching in the parameter space the direction $\delta \theta_{k}=-\partial D / \partial \theta_{k}$ of more rapid change; for this, an iterative procedure ${ }^{[17]}$ 


$$
\theta_{k}^{n+1}=\theta_{k}^{n}+\lambda^{2} \sum_{i}\left(y_{i}-f(x, \theta)\right)\left(\frac{\partial f_{i}}{\partial \theta_{k}}\right)_{\theta=\theta^{n}},
$$

where $\lambda$ is a scaling parameter, can be followed until the desired convergence condition

$$
\sum_{i=1}^{N}\left|D^{i+1}\left(\theta_{k}\right)-D^{i}\left(\theta_{k}\right)\right|<\epsilon
$$

is satisfied. Compared to other minimization schemes, this so-called steepest-descent method ${ }^{26]}$ of nonlinear fitting has an inherent greater simplicity and demands a shorter computational time.

In all examples discussed below the steepest-descent algorithm was used to determine the values of the $\left\{f_{n}, \gamma_{n}\right\}$ parameters which best fit the theoretical expressions of the reflectance (see Section II) to a given experimental spectrum. Once identified, this set of best parameters was adopted to allow the numerical simulation the spectra of other yet to be performed experiments.

\subsection{Applications}

\subsubsection{Isotropic films of behenic acid}

\begin{tabular}{|c|c|c|c|}
\hline Peak & Assignment & force constant & width \\
\hline $\bar{\nu}_{n}\left(\mathrm{~cm}^{-1}\right)$ & & $f_{n}\left(a m u^{-1}\right)$ & $\gamma_{n}\left(\mathrm{~cm}^{-1}\right)$ \\
\hline 1686.4 & $(\mathrm{C}=\mathrm{O}) \nu_{s}$ & 0.0216 & $2.35481 \times 10^{1}$ \\
1700.8 & $(\mathrm{C}=\mathrm{O}) \nu_{a}$ & 0.0595 & $2.25772 \times 10^{1}$ \\
2572.9 & $\mathrm{OH}_{d e f} \cdots \mathrm{CO} \nu$ & 0.1481 & $3.23660 \times 10^{2}$ \\
2676.9 & $\mathrm{OH}_{d e f} \cdots \mathrm{CO} \nu$ & 0.1591 & $2.92913 \times 10^{2}$ \\
2847.8 & $\left(\mathrm{CH}_{2}\right) \nu_{s}$ & 0.0638 & $8.18667 \times 10^{0}$ \\
2871.6 & $\left(\mathrm{CH}_{3}\right) \nu_{s}$ & 0.1143 & $8.63443 \times 10^{1}$ \\
2915.3 & $\left(\mathrm{CH}_{2}\right) \nu_{a}$ & 0.1779 & $1.65968 \times 10^{1}$ \\
2953.9 & $\left(\mathrm{CH}_{3}\right) \nu_{a}$ & 0.0061 & $6.36851 \times 10^{0}$ \\
3026.5 & $(\mathrm{OH} \cdots \mathrm{O}) \nu$ & 0.2084 & $3.63072 \times 10^{2}$ \\
\hline
\end{tabular}

Table 1: Principal features of the ATR spectrum of a isotropic thick film of behenic acid deposited on a ZnSe crystal and corresponding force constant and width parameters obtained through a best-fitting procedure. 


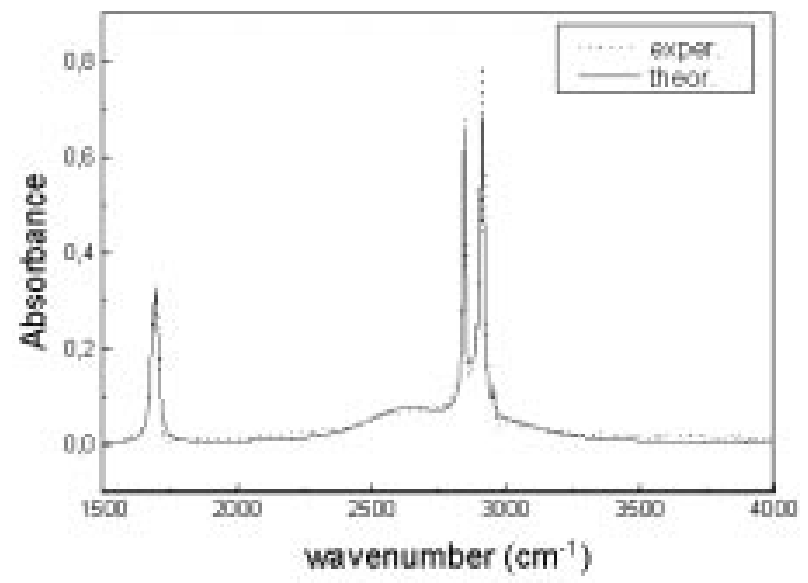

Figure 5. Comparison between the experimental (dotted line) and best-fitted (continuous line) $45^{\circ}$ ATR spectra of a thick film of behenic acid deposited on top of a ZnSe crystal.

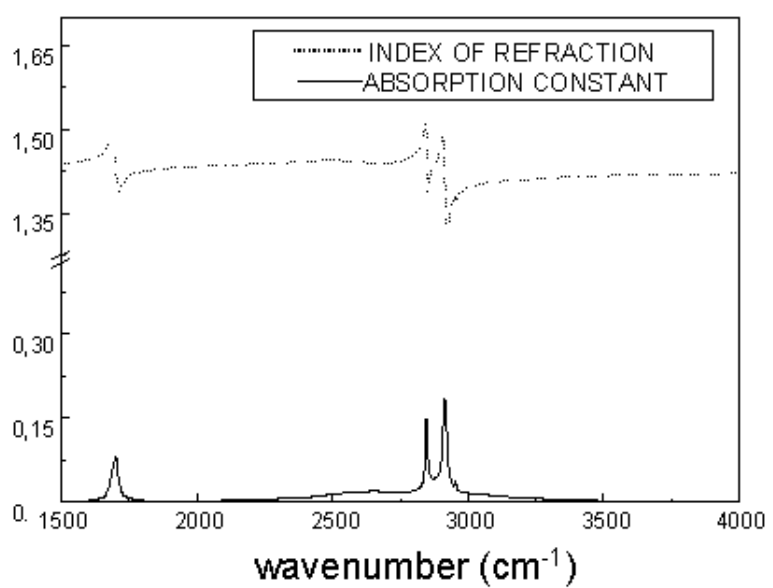

Figure 6. Predicted behavior in the IR region for the index of refraction $n(\bar{\nu})$ and absorption coefficient $k(\bar{\nu})$ of a thick film of behenic acid.

Experimental: All infrared spectra were collected with a $4 \mathrm{~cm}^{-1}$ resolution using a MB-100 FTIR spectrometer (Bomem, Canada) equipped with a deuterated triglycine sulfate (DTGS) detector. For the ATR experiments we have used a ZnSe $\left(n_{1}=2.42\right)$ crystal (Harrick Scientific, USA) with $45^{\circ}$ faces mounted in an appropriate accessory (Specac, UK), and 256 scans were collected to produce each spectrum.

The isotropic film was prepared by dripping a solution of b.a. $\left(d=0.8221 \mathrm{~g} / \mathrm{cm}^{3}\right.$, index of refraction $n=1.43$ and molecular weight equal to $340.57 \mathrm{Da}$, obtained from Sigma, USA) in a volatile solvent (chloroform) on top of the ZnSe crystal, until no further increase in the intensity could be observed in the ATR spectrum. (This would assure that the film thickness is larger than the penetration length of the radiation.)
As indicated in Table 1, the peaks were assigned ${ }^{[28]}$ respectively to the $i)$ symmetric $\left(\nu_{s}\right)$ and asymmetric $\left(\nu_{a}\right) C=O$ stretchings $\left(1686.4 \mathrm{~cm}^{-1}\right.$ and $\left.1700.8 \mathrm{~cm}^{-1}\right)$, ii) symmetric $\left(\nu_{s}\right)$ and asymmetric $\left(\nu_{a}\right) \mathrm{CH}_{2}$ stretchings $\left(2847.8 \mathrm{~cm}^{-1}\right.$ and $\left.2915.3 \mathrm{~cm}^{-1}\right)$, iii) symmetric $\left(\nu_{s}\right)$ and asymmetric $\left(\nu_{a}\right) \mathrm{CH}_{3}$ stretchings $\left(2871.6 \mathrm{~cm}^{-1}\right.$ and $\left.2953.9 \mathrm{~cm}^{-1}\right)$, iv) coupling of the $O H$ deformation mode to the symmetric $\left(\nu_{s}\right)$ and asymmetric $\left(\nu_{a}\right) \mathrm{CH}_{3}$ stretchings $\left(2572.9 \mathrm{~cm}^{-1}\right.$ and $\left.2676.9 \mathrm{~cm}^{-1}\right)$, and, finally, $v$ ) the $O H \cdots H$ hydrogen bond stretching $\left(3026.5 \mathrm{~cm}^{-1}\right)$.

Numerical fitting of the ATR spectrum: With basis on the above data, the number of particles per volume unit of the fatty acid was calculated $(N=$ $1.45 \times 10^{21} \mathrm{~cm}^{-3}$ ) and the electronic contribution to the polarizability estimated as $\alpha_{0}=6.13 \times 10^{-2} \mathrm{~cm}^{3}$. For the spectral simulation of a thin film deposited on a steel plate, we adopted the values ${ }^{[29]} d=7.86 \mathrm{~g} / \mathrm{cm}^{3}$ and an atomic weight of $55.85 \mathrm{Da}(\mathrm{Fe})$ to describe the substrate - with a consequent estimated plasma frequency $\varpi_{p}=1.43 \times 10^{21} s^{-1}$.

Since the incoming radiation was assumed not to be polarized, we have considered an average between the perpendicular and parallel reflectance expressions (Eqs. (1)-(2)). The fitting of the experimental ATR spectrum in the $(1500-4000) \mathrm{cm}^{-1}$ interval was implemented using the steepest-descent algorithm. At convergence which was achieved with a final residue $\epsilon \sim 10^{-2}$ - the optimized parameters $f$ and $\gamma$ were those shown in Table 1.

Theoretically predicted spectra: The values of $n(\bar{\nu})$ and $k(\bar{\nu})$ were obtained (Fig. 6) directly from Eq. (8), and then it becomes a simple matter to simulate the theoretical spectra of the material in different physical situations. Let's consider first how the variation of the incidence angle $\Phi$ affects the ATR spectra of the isotropic b.a. film. As it can be seen in Fig. 7, the larger SNR is obtained for an incidence angle close to the critical value of $35^{\circ}$. While for a lower value $\left(\Phi=10^{\circ}\right)$ we obtain a typical external reflection spectrum with low $\mathbf{S N R}^{[30]}$, for $\Phi=60^{\circ}$ there is strong absorption; it is instructive to note the similarity of the ATR spectra in these two limits to the predicted behavior of $n$ and $k$ (Fig. 6), respectively. 


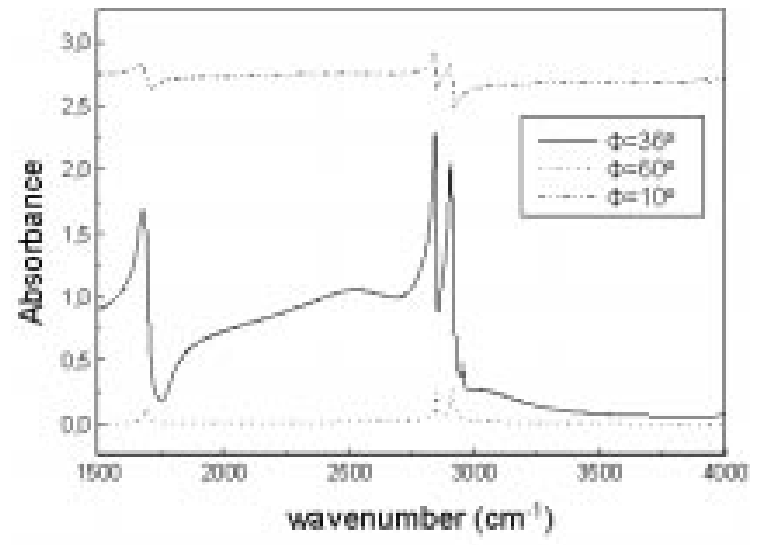

Figure 7. Numerically simulated ATR spectra of a thick film of behenic acid deposited on a ZnSe crystal, for different incidence angles $\Phi$.

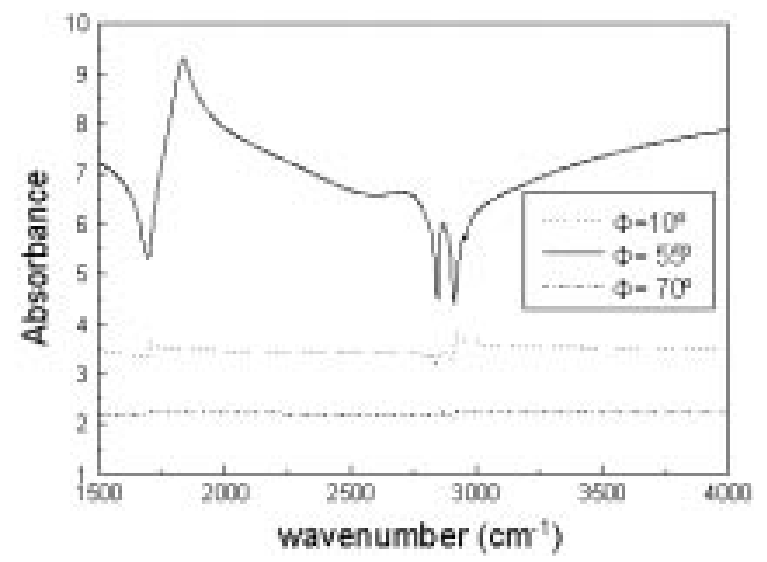

Figure 8. Numerically simulated external reflection spectra of a thick film of behenic acid deposited on a ZnSe crystal, for different incidence angles $\Phi$. At values of $\Phi \sim \Phi_{B}=55^{\circ}$ the $\mathbf{P}$-component of the radiation is strongly absorbed (see Fig. 2).

As we have discussed in Section IIA, in an external reflection experiment the absorbance of the $\mathbf{P}$-polarized (i.e., along the direction parallel to the incidence plane) radiation should go through a maximum at the Brewster angle. In Fig. 8 we observe that in fact the simulated erm spectra exhibit this peculiar behavior at $\Phi=55^{\circ}$.

Let's now consider the case of a very thin $(d \sim \AA)$ but still isotropic film of b.a. deposited on a metallic substrate. As depicted in Fig. 9, for increasing values of $\Phi$ there is a noticeable reduction in the intensities of the absorption bands, in agreement to the expected behavior at the grazing angle limit (see Section IIA). Although we do not show the corresponding result here, the simulation also reveals that for a thin film on a metallic substrate there is no absorption of the $\mathbf{S}$-component of the radiation. This is in agreement with the well-known observation that while the ga configuration is specially adequate to the inves-

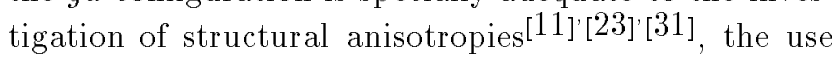
of $\mathbf{P}$-polarized radiation contributes to a much better SNR.

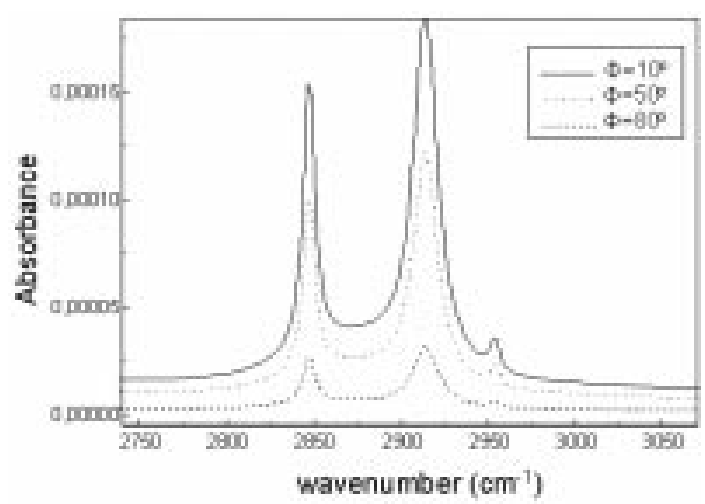

Figure 9. Numerically simulated external reflection spectra of a thin $(d \sim \AA)$ film of behenic acid deposited on a metallic substrate, for different incidence angles $\Phi$.

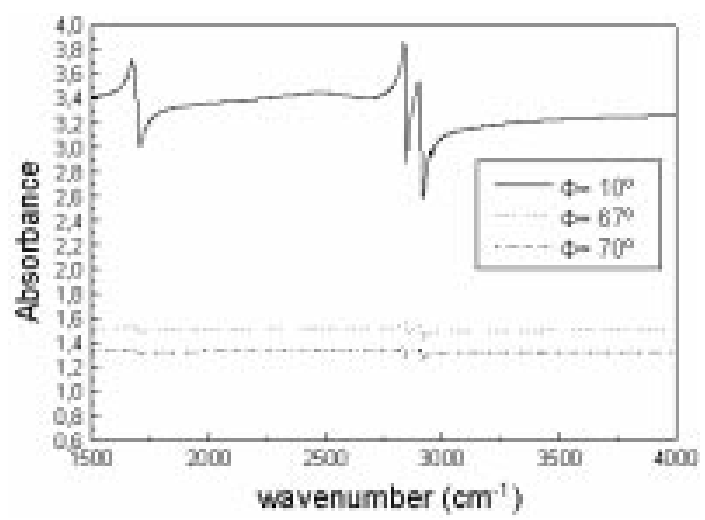

Figure 10. Numerically simulated external reflection spectra of a thin $(d \sim \AA)$ film of behenic acid deposited on a $\mathrm{ZnSe}$ substrate, for different incidence angles $\Phi$ of a non-polarized IR radiation.

Finally, we will consider the case of a external reflection experiment for a thin film of b.a. on top of a $\mathrm{ZnSe}$ crystal. Once again one can observe that a progressive reduction in the intensities of the absorption bands is predicted with the increase of the incidence angle $\Phi$ (see Fig. 10). The different reflective properties of a thin film for the $\mathbf{S}$ - and $\mathbf{P}$-components of the incident radiation are evident in Fig. 11, where we represent the erm $\Phi=10^{\circ}$ simulated spectra for the individual polarizations: while the $\mathbf{P}$-spectrum exhibits both positive and negative absorption bands, only negative bands can be observed in the $\mathbf{S}$-spectrum. (Again, one should observe the similarities of each one these two curves to either the $n$ or the $k$ behavior presented in Fig. 6.) 


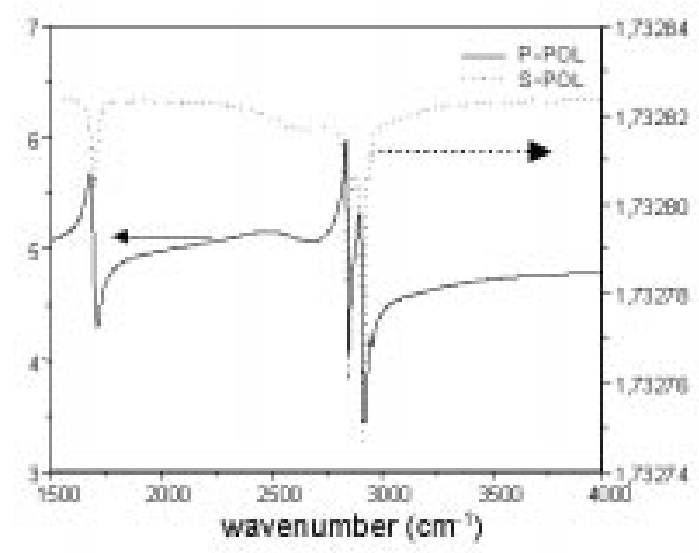

Figure 11. Numerically simulated external reflection spectra of a thin $(d \sim \AA)$ film of behenic acid deposited on a $\mathrm{ZnSe}$ substrate, for an incidence angle $\Phi=10^{\circ}$ of $\mathbf{S}$ - (dashed line) and $\mathbf{P}$ - (continuous line) polarized IR radiation. Please note that the absorption intensities for the $\mathbf{P}-$ component are much larger than those of the $\mathbf{S}$ - radiation, and that therefore the $\mathbf{P}$-polarization must dominate the corresponding $\Phi=10^{\circ}$ spectrum in Fig. 10 .

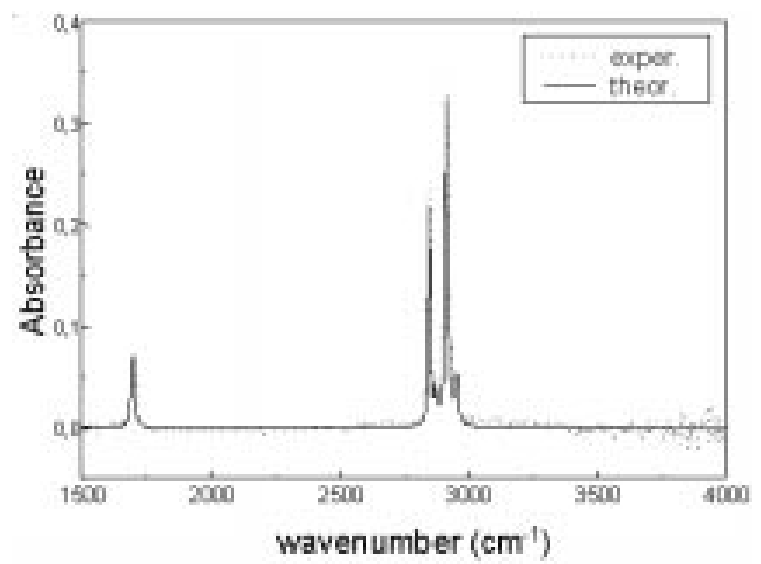

Figure 12. Comparison between the experimental (dotted line) and best-fitted (continuous line) S- polarized ATR spectra, at an incidence angle of $45^{\circ}$, of a LB film of behenic acid deposited on top of a ZnSe crystal.

\subsubsection{LB films of behenic acid}

The spectral simulation procedure will now be used to establish how the IR spectroscopic properties of a thin isotropic LB film of a fatty acid will depend on the sample thickness $d$.

Experimental: Behenic acid was used as received (Sigma, USA) to prepare LB films by the careful spreading of $100 \mu \mathrm{L}$ of a chloroform solution the fatty acid on an air-water interface in a LB-5000 Langmuir trough (KSV, Finland). Ultra-pure (resistivity greater than $16 \mathrm{M} \Omega / \mathrm{cm}$ ) water obtained after passage through a Nanopure system (Barnstead, USA) was used as sub-phase. The compression of the floating monolayer was achieved with a $10 \mathrm{~mm} / \mathrm{min}$ moving nylon barrier, until a surface pressure of $35 \mathrm{mN} / \mathrm{m}$ was reached. Then, using backward and forward barrier speeds of $2.0 \mathrm{~mm} / \mathrm{min}$, the transfers were performed at $25^{\circ} \mathrm{C}$ always in the upstroke movement (dipping speed equal to $4.0 \mathrm{~mm} / \mathrm{min}$ ) of a ZnSe substrate, resulting in X-type LB films ${ }^{[2]}$.

A homemade accessory was used to hold the ZnSe crystal for the ATR study, and the same spectroscopic conditions as described above were used to collect the spectral data. In Table 2, we list the principal features identified in the ATR spectrum of the thin film, where assignments in common to those of Table 1 were as previously discussed.

\begin{tabular}{|c|c|c|c|}
\hline Peak & Assignment & force constant & width \\
\hline $\bar{\nu}_{n}\left(\mathrm{~cm}^{-1}\right)$ & & $f_{n}\left(\mathrm{amu}^{-1}\right)$ & $\gamma_{n}\left(\mathrm{~cm}^{-1}\right)$ \\
\hline 1686.4 & $(\mathrm{C}=\mathrm{O}) \nu_{s}$ & 0.0012 & $1.0448 \times 10^{1}$ \\
1700.8 & $(\mathrm{C}=\mathrm{O}) \nu_{a}$ & 0.0060 & $1.2530 \times 10^{1}$ \\
2847.8 & $\left(\mathrm{CH}_{2}\right) \nu_{s}$ & 0.0200 & $8.0031 \times 10^{0}$ \\
2871.6 & $\left(\mathrm{CH}_{3}\right) \nu_{s}$ & 0.0048 & $1.2782 \times 10^{1}$ \\
2915.3 & $\left(\mathrm{CH}_{2}\right) \nu_{a}$ & 0.0471 & $1.2203 \times 10^{1}$ \\
2953.9 & $\left(\mathrm{CH}_{3}\right) \nu_{a}$ & 0.0086 & $1.4926 \times 10^{1}$ \\
\hline
\end{tabular}

Table 2: Principal features of the ATR spectrum of a LB film of behenic acid deposited on a ZnSe crystal and corresponding force constant and width parameters obtained through a best-fitting procedure. 
Numerical fitting of the ATR spectrum: The number of particles per volume unit of the b.a., $N$, and the electronic contribution to the polarizability, $\alpha_{0}$, were the same as before. In the simulation of the grazing angle spectra, the parameters corresponding to a steel plate ${ }^{[29]}$ were once again adopted for the description of the metallic reflective element. In Table 2 we also show the parameters $f$ and $\gamma$ which best fit the experimental ATR spectrum in the $(1500-4000) \mathrm{cm}^{-1}$ after the steepest-descent algorithm was used. Once again a final residue $\epsilon \sim 10^{-2}$ was found at the convergence limit of the numerical procedure. The quality of the fitting can be appreciated in Fig. 12, where the experimental and adjusted spectra are compared.

Theoretically predicted spectra: With the optimized set of parameters $f$ and $\gamma$ we have used Eq. (8) to generate the $n(\bar{\nu})$ and $k(\bar{\nu})$ functions (Fig. 13), a necessary step for the numerical simulation of the different desired spectra. Let's examine first how the thickness of the film influences its ATR spectrum. In Fig. 14 we show the corresponding $\Phi=45^{\circ}$ spectra for different values of the thickness $d$. As it can be observed, at this value of the incidence angle the intensities of the characteristic absorbance peaks change only moderately as a function of $d$; this fact then precludes the use of the ATR spectroscopy as a reliable technique to determine the thickness of LB films of organic compounds. This result can be understood if we remember the same characteristics which make the ATR spectroscopy a good technique to probe interfaces reduce its sensitiveness to bulk properties.

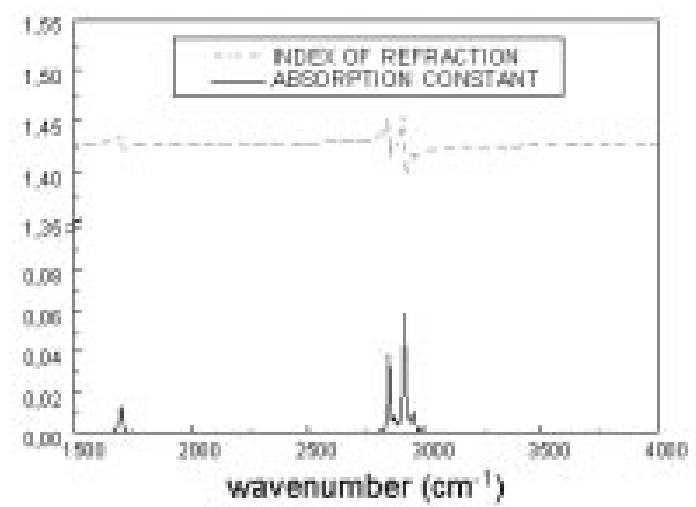

Figure 13. Predicted behavior for the index of refraction $n(\bar{\nu})$ and the absorption coefficient $k(\bar{\nu})$ of a LB film of behenic acid deposited on top of a ZnSe crystal.

A more interesting point comes out when we adopt a configuration such that the incidence angle is lower than the critical value ( $\Phi_{c}=36^{\circ}$ for b.a. on ZnSe).
In Fig. 15 we present the predicted $\Phi=30^{\circ}$ spectra in the limiting cases of very thin and very thick films. One can observe that while for the thick sample the spectrum is similar to an external reflection (since $\Phi<\Phi_{c}$ ), for the very thin film the same ATR profile as before is found. This is a consequence of the fact that for very thin samples the critical angle becomes determined by the ratio between the indices of refractions of the third medium (air) and of the ire (and, therefore, in this case it is equal to $24.4^{\circ}$ ).

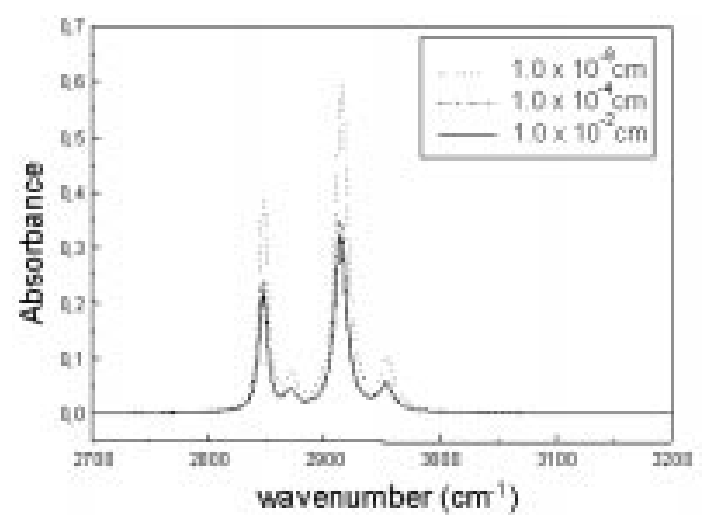

Figure 14. Numerically simulated ATR spectra of a LB film of behenic acid deposited on a ZnSe crystal, for different values of the thickness $d$.

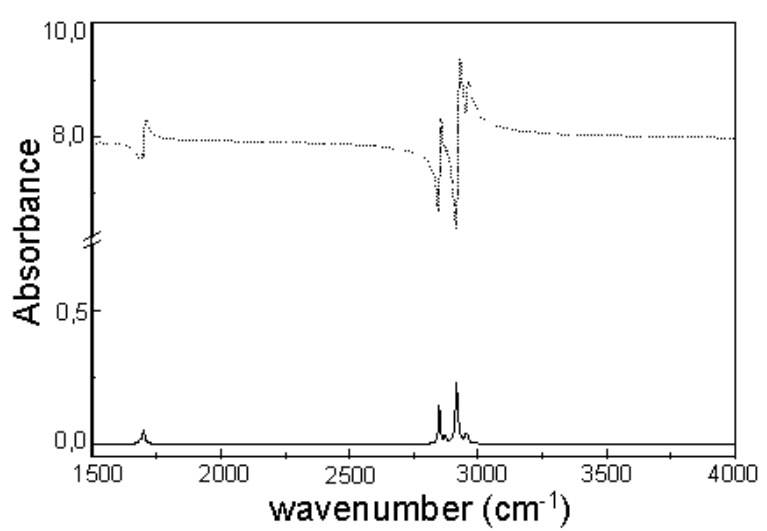

Figure 15. Numerically simulated $\Phi=30^{\circ} \mathrm{irm}$ spectra of a LB film of behenic acid deposited on a ZnSe crystal, for the limiting cases $d=1.0 \times 10^{-8} \mathrm{~cm}$ (continuous line) and $d=1.0 \times 10^{2} \mathrm{~cm}$ (dashed line).

As a final example, we consider the influence of the thickness $d$ upon the absorption intensities in the grazing angle spectra of LB films. The numerical results indicate that while at the two extreme limits of very thin or very thick films the intensities of the characteristic absorption peaks at $2916 \mathrm{~cm}^{-1}\left(\left(\mathrm{CH}_{2}\right) \nu_{a}\right)$ and $1700 \mathrm{~cm}^{-1}\left((\mathrm{C}=\mathrm{O}) \nu_{a}\right)$ do not change in a noticeable way with the thickness variation, at the intermediate 
$10^{-5} \mathrm{~cm} \lesssim d \lesssim 10^{2} \mathrm{~cm}$ range the slopes of the absorption intensity curves are such as (see Fig. 16) to suggest that the ga configuration could be adequate for the determination of the thickness of LB films. However, the presence of interference fringes associated to the multiple internal reflections at the two interfaces of the film limit this range of applicability to the $10^{-5} \mathrm{~cm} \lesssim d \lesssim 10^{-2} \mathrm{~cm}$ region. Note that for the system considered, the best SNR is predicted to occur for values of $d \sim 10 \mu \mathrm{m}$, a thickness characteristic of spin coated or evaporated films.

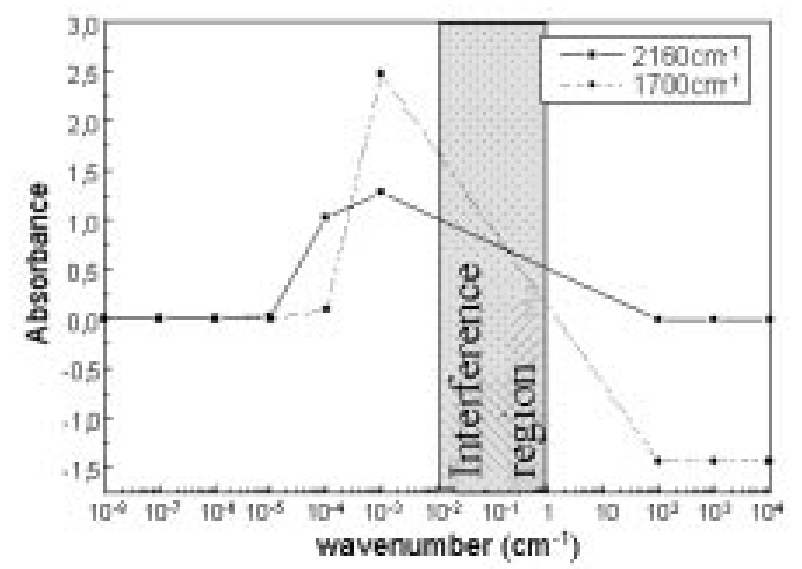

Figure 16. Predicted variation of the absorption intensities of the $2916 \mathrm{~cm}^{-1}$ and $1700 \mathrm{~cm}^{-1}$ peaks of a $b . a$. film deposited on top of a metal surface, obtained from the numerically simulated grazing angle spectrum.

\section{Conclusion}

In this paper we have examined how the numerical simulations of the infrared spectra of organic films can be used to investigate the characteristics of different spectroscopic experiments prior to their actual realization. With this, the range of applicability and eventual limitations of each experimental IR configuration can be thoroughly investigated in a purely theoretical and simple and economical manner. As a consequence, for a given problem under consideration one can then select to perform only the most promising and adequate experiments.

We began by showing how the optical constants of the material could be obtained from a simple model of independent harmonic oscillators for its normal modes. At the same time, from the Fresnel equations - which result directly from the application of the boundary conditions obeyed by the electromagnetic radiation at the interfaces of the sample - the reflectance spectra corresponding to different experimental configurations of interest were derived in terms of the optical constants of the sample and of any other media probed by the radiation. Then, after a given experimental spectrum (for instance, ATR at a given incidence angle $\Phi$ ) was selected, we have used a straightforward numerical minimization routine to best-fit the corresponding theoretical expression, obtaining in this way the correct estimate for the behavior of the index of refraction $n(\bar{\nu})$ and absorption constant $k(\bar{\nu})$ of the material. Once these functions were known, the spectrum of any other experimental configuration could be numerically simulated.

As examples of the applicability of the technique, we have presented results for two systems of interest: i) relatively thicker evaporated (and therefore isotropic) and ii) thin Langmuir-Blodgett prepared (and hence intrinsically organized) films of behenic acid. In each case, the ATR spectra of b.a. films deposited in ZnSe crystals were used to optimize the force constant and width parameters of the relevant absorption features of the material; then, the functions $n(\bar{\nu})$ and $k(\bar{\nu})$ were obtained, from which the corresponding spectra for several different possible IR configurations were predicted. For the case of LB films of behenic acid, in special, an analysis of the possible use of IR spectroscopic techniques as a convenient tool for the determination of the thickness of the samples was presented.

Since LB films of fatty acids are prototypal of organized thin organic films, it is expected that studies similar to that presented here could find general application in the selection of the best suited IR geometry to investigate the spectroscopic properties of many similar systems, such as self-assembled multilayers or conjugated polymer samples in general. For instance, we should point out that recently reflectance studies have become an invaluable complementary tool for the characterization of the transport properties of conducting polymers $^{[32]}$.

Also, one can incorporate the use of polarized radiation in the theoretical treatment above discussed and, hence, numerical simulation of the infrared spectra will become of particular importance in the study of the structural order in organized molecular films. As an example of a problem where this technology can be applied, we single out the comparison between the degree of microscopic organization of self-assembled and Langmuir-Blodgett films of similar polymeric systems. This is a question of urgent interest due to the need to establish the relative molecular orientation in thin 
organic films, which are nowadays finding applications in different branches of scientific investigations.

\section{Acknowledgments}

We acknowledge the financial support of the Brazilian agencies FINEP, CNPq and FACEPE.

\section{References}

[1] G. L. Gaines, Insoluble Monolayers at Liquid Gas Interface (Wiley, New York, 1990).

[2] M. C. Petty, Langmuir-Blodgett Films: An Introduction (Cambridge University Press, Cambridge, 1996).

[3] G. Roberts, Langmuir-Blodgett Films (Plenum Press, New York, 1990).

[4] R. H. Tredgold, Order in Thin Organic Films (Cambridge University Press, Cambridge, 1994).

[5] G. Decher, J. D. Hong and J. Schmitt, Thin Solid Films 210-211, 831 (1992).

[6] M. Ferreira, O. Onitsuka, A. C. Fou, M. F. Rubner and B. Hsieh, J. Appl. Phys. 79, 7501 (1996).

[7] C. P. de Melo, C. G. dos Santos, F. L. dos Santos, M. V. dos Santos and J. E. G. de Souza, work in progress.

[8] See, for example, J. Majewski, T. L. Kuhl, M. C. Gerstenberg, J. N. Israelachvili and G. S. Smith, J. Phys. Chem. B 101, 3122 (1997), and references therein.

[9] D. J. Ahn and E. I. Frances, J. Phys. Chem. 96, 9952 (1992).

[10] N. B. Colthup, Introduction to Infrared and Raman Spectroscopy, (Academic Press, New York, 1975).

[11] C. P. de Melo and M. I. Mosquera-Sánchez, Langmuir 14, 490 (1998).

[12] A.C. Tenório and C.P. de Melo, Proceedings of the International Conference on Science and Technology of Synthetic Metals (ICSM'98), July 1998, Montpellier, França.

[13] M. Milosevic and S., L. Berets, Appl. Spectroscopy 47, 566 (1993).

[14] J. D. Jackson, Classical Electrodynamics (Wiley, New York, 1962).
[15] F. M. Mirabella, Jr., Appl. Spectrosc. 21, 45 (1985).

[16] T. Hasegawa, J. Umemura and T. Takenaka, J. Phys. Chem. 97, 9009 (1993).

[17] A. C. Tenório, Determinação da Orientação Molecular em Filmes Orgânicos Finos por Espectroscopia na Região do Infravermelho, MSc thesis presented to the Departamento de Física, Universidade Federal de Pernambuco, Recife, Brazil, December 1997.

[18] J. R. Reitz, F. J. Milford and R. W. Christy, Foundations of Electromagnetic Theory (Addison-Wesley, London, 1979).

[19] J. F. Rabolt, M. Jurich and J. D. Swalen, Applied Spectrosc. 39, 269 (1984).

[20] R. A. Dluhy, N. A. Wright and P. R. Griffiths, Applied Spectrosc. 42, 138 (1988).

[21] C. Kittel, Introduction to Solid State Physics (John Wiley, New York, 1996).

[22] S. A. Francis and A. H. Ellington, J. Opt. Soc. Am. 49, 31 (1959).

[23] C.G. dos Santos, C.P. de Melo and R. Souto Maior, Synthetic Met. 71, 2083 (1995).

[24] N. J. Harrick, Internal Reflection Spectroscopy (Harrick Scientific, New York, 1987).

[25] E. B. Wilson, Jr., J. C. Decius and P. C. Cross, Molecular Vibrations (McGraw-Hill, New York, 1955).

[26] P. K. Mackeown and D. J. Newman, Computational Techniques in Physics (McGraw-Hill, New York, 1971).

[27] D. C. Harris and M. D. Bertolucci, Symmetry and Spectroscopy (Dover, New York, 1989).

[28] C. J. Pouchert, The Aldrich Library of FTIR Spectra Vol I (Aldrich Chemical Company Inc., Milwauke, 1989).

[29] The Merck Index (Merk \& CO Inc., Rachway, 1989).

[30] E. Hecht and A. Zajac, Optics (Addison-Wesley, London, 1974).

[31] J. D. Swalen, J. Mol. Electron. 2, 155 (1986).

[32] K. Lee, A. J. Heeger and Y. Cao, Synthetic Met. 72, 25 (1995). 